This item was submitted to Loughborough's Research Repository by the author.

Items in Figshare are protected by copyright, with all rights reserved, unless otherwise indicated.

\title{
Kinetics of spreading of synergetic surfactant mixtures in the case of partial wetting
}

PLEASE CITE THE PUBLISHED VERSION

http://dx.doi.org/10.1016/j.colsurfa.2015.11.026

\section{PUBLISHER}

(C) Elsevier

VERSION

AM (Accepted Manuscript)

\section{PUBLISHER STATEMENT}

This work is made available according to the conditions of the Creative Commons Attribution-NonCommercialNoDerivatives 4.0 International (CC BY-NC-ND 4.0) licence. Full details of this licence are available at: https://creativecommons.org/licenses/by-nc-nd/4.0/

\section{LICENCE}

CC BY-NC-ND 4.0

\section{REPOSITORY RECORD}

Kovalchuk, Nina, Faiz M. Mahdi, Victor Starov, and Anna Trybala. 2016. "Kinetics of Spreading of Synergetic Surfactant Mixtures in the Case of Partial Wetting". figshare. https://hdl.handle.net/2134/23212. 


\title{
Kinetics of spreading of synergetic surfactant mixtures in the case of partial wetting.
}

\author{
Nina Kovalchuk ${ }^{\mathrm{a}, \mathrm{b}}$, Anna Trybala ${ }^{\mathrm{a}}$, Faiz Mahdi ${ }^{\mathrm{a}}$, Victor Starov ${ }^{\mathrm{a}^{*}}$ \\ ${ }^{\text {a} D e p a r t m e n t ~ o f ~ C h e m i c a l ~ E n g i n e e r i n g, ~ L o u g h b o r o u g h ~ U n i v e r s i t y, ~}$ \\ Loughborough, LE 11 3TU, UK \\ ${ }^{\mathrm{b}}$ Institute of Biocolloid Chemistry, 42 Vernadsky av. Kiev,03142, Ukraine
}

\begin{abstract}
Spreading kinetics of mixtures of hydrocarbon surfactant, octaethylene glycol monododecyl ether, and fluoro-surfactant, Zonyl FSN-100, on highly hydrophobic substrate was experimentally studied. The mixtures reveal a synergism in their wetting properties with equilibrium contact angle of mixtures being 15-20 ${ }^{\circ}$ lower than that of individual surfactant solutions. The synergism is due to different affinity of the surfactants to the liquid/air and liquid/solid interface. Both individual surfactants and their mixtures demonstrate power law kinetics of spreading over the time span of tens of seconds. The spreading exponent is lower than that for pure liquids, but spreading exponent of mixtures is higher than that of individual solutions. The maximum in the spreading exponent is observed for the mixtures demonstrating the lowest equilibrium contact angles. For these mixtures the spreading exponent is close to that of pure liquids.
\end{abstract}

Keywords: oxiethylated alcohol $\mathrm{C}_{10} \mathrm{EO}_{8}$, fluoro-surfactant Zonyl FSN-100, sylanized glass substrate, interfacial tension, equilibrium contact angle, spreading exponent.

*Corresponding author: V.M.Starov@lboro.ac.uk 


\section{Introduction}

Spreading kinetics of various liquids on solid surfaces is of great importance in many industrial applications including painting, printing, coating etc. The problem has been studied thoroughly for the case of pure liquids. Spreading kinetics in the case of complete wetting consists of two stages. During the first, inertial stage, radius of spread drop increases proportionally to the square root of time, $R \sim \sqrt{t}[1,2]$. The duration of this stage is in the range of millisecond, with characteristic timescale given by $\left(\rho R^{3} / \gamma\right)^{1 / 2}$. Later viscous dissipation becomes more important than inertia and spreading slows down. In the purely viscous regime, when inertia is negligible $R \sim t^{0.1}[3,4]$. For the droplets larger than the capillary length $\left(l_{c}=\sqrt{\frac{\gamma}{\rho g}}\right)$, the spreading exponent increases to $\alpha=0.125$, due to contribution of gravity [5].

In the case of partial wetting by pure liquids both pre-factor and spreading exponent during the inertial stage of spreading decrease with the increase of the equilibrium contact angle [6]. As usually a static advancing contact angle is referred to below as "equilibrium contact angle". Viscous stage of wetting was observed only for liquids with small contact angles with threshold value of equilibrium contact angle increasing with an increase of the liquid viscosity [2]. According to [2] for the mixtures of water and glycerol with viscosity below $35 \mathrm{mPa} \cdot \mathrm{s}$ only inertial stage of spreading was observed on substrates with contact angle $63^{\circ}$. The spreading in those cases was completed within approximately $10 \mathrm{~ms}$. For the mixtures with viscosity of $35 \mathrm{mPa} \cdot \mathrm{s}$ and above the viscous stage was observed on the same substrate. The spreading exponent was $\alpha \sim 0.1$, i.e. similar to that of complete wetting case [2].

Very often a liquid to spread is an aqueous formulation. Surfactants are the common additives improving spreading characteristics of such formulations. In the case of complete wetting surfactant solutions demonstrate initially the same two stages as pure liquids, inertial and viscous [7]. According to [7] spreading during the inertial stage does not depend on surfactant properties and concentration and is similar to that of pure liquids, whereas during the viscous stage it becomes surfactant depending and spreading exponent may be higher than that for pure liquids [7]. The most important distinction of the spreading of surfactant solutions from that of pure liquids in the case of complete wetting is the third stage, referred to as surfactant 
enhanced spreading or superspreading [7-15]. During this stage the spreading accelerates again demonstrating spreading exponent up to 0.5 . The precise mechanism governing this stage is not completely established yet. The available experimental results and most promising hypotheses on mechanism of surfactant enhanced spreading and superspreading are discussed in recent reviews [16-18].

In the case of partial wetting the spreading of surfactant solutions depends on the equilibrium contact angle similar to pure liquids. For conventional surfactants on highly hydrophobic substrates (large equilibrium contact angles) only inertial stage of spreading was observed [7], whereas for surfactant solutions with small equilibrium contact angle it was followed by the viscous stage with spreading exponent $\alpha \sim 0.1$. For example the viscous stage was observed in [7] for solution of trisiloxane surfactant TSS 10/2 on polypropylene (equilibrium contact angle $\sim 12^{\circ}$ ).

The experiments in [7] have been performed on rather short time scale to catch the early kinetics, maximum time of observation was $10 \mathrm{~s}$. The study presented in [19] has been devoted the kinetics of partial wetting of surfactant solutions at time scale of tens of seconds. In agreement with [2, 7] ionic surfactants on highly hydrophobic surfaces, such as Teflone, Parafilm and polypropylene (advancing contact angle of water $\mathrm{CAW} \geq 99^{\circ}$ ), demonstrated constant value of equilibrium contact angle at $t>1 \mathrm{~s}$ (i.e. spreading was finished on much shorter time scale). On substrates of higher energy, poly(vinylfluoride), $\mathrm{CAW}=78^{\circ}$, and polyethyleneterephthalate, $\mathrm{CAW}=77^{\circ}$, spreading continued during much longer, up to $30 \mathrm{~s}$ and duration increased with an increase of concentration. Non-ionic surfactant, pentaethylene glycol monododecyl ether, $\mathrm{C}_{12} \mathrm{EO}_{5}$, demonstrated slow longtime spreading kinetics on all substrates studied in [19] at concentrations above $0.25 \mathrm{cmc}$.

It should be emphasized that the spreading exponent at $t>1 \mathrm{~s}$ according to [19] was smaller than 0.1 , that is surfactant solutions in this case spread slower than pure liquids. This small value have been ascribed in [19] to adsorption of surfactant onto solid/liquid and solid/air interface. The theoretical basis of the slow spreading of surfactant solutions due to surfactant adsorption on the solid/air interface in the front of contact line was proposed in [20,21]. Experimental evidence of such adsorption was provided in [22].The slow spreading of long duration was found also in [23], for 
several surfactant solutions, both ionic and non-ionic. Kinetics of spreading in this case was in good agreement with the theory developed in [20, 21].

Despite the numerous studies on the wetting properties of surfactant solutions, and in particular on partial wetting, the precise values of the spreading exponents during the slow stage of spreading and their dependence on the equilibrium contact angle to the best of our knowledge were not addressed in previous studies. Therefore the aim of the present study is to fill this gap.

The wetting performance of any liquid, including surfactant solution can be estimated using the Young equation:

$\cos \theta=\frac{\sigma_{s v}-\sigma_{s l}}{\sigma_{l v}}$

where $\theta$ is the contact angle, $\sigma_{s v}$ is the solid-vapor surface tension (specific surface energy od solid), $\sigma_{s /}$ is the solid-liquid surface tension and $\sigma_{l v}$ is the liquid-vapor surface tension. The partial wetting correspond to the case $1>\cos (\theta)>0$ (i.e. $0<\theta<90^{\circ}$ ) and the larger is $\cos (\theta)$ the better are the wetting properties. It follows from the Eq. (1) that the improvement of wetting properties is possible by decreasing $\sigma_{s /}$ and/or $\sigma_{l v}$. The decrease can be achieved by adding surfactants to a wetting liquid. Note, by using of surfactant mixtures the liquid-vapor surface tension cannot be the only criterion to predict spreading performance, because affinity of various components of mixtures to various surfaces can be different. One of such cases is considered below.

To provide the systematic changes in the contact angle we used synergetic mixtures of two surfactants [24], both at concentrations above critical micellar concentration (cmc). One of the surfactants is fluorinated. According to the literature (see [25] and references herein) fluorosurfactants adsorb readily on water/air interface lowering the surface tension of aqueous solutions to values $\sim 20 \mathrm{mN} / \mathrm{m}$, but their adsorption on hydrocarbon surfaces is much lower. That is why their spreading performance is much worse than it can be expected based on the surface tension value. The wetting properties can be improved by mixing fluorosurfactants with conventional hydrocarbon surfactants. It should be emphasized that despite proven industrial applications of synergetic mixtures of fluoro- and hydrocarbon surfactants (for example, in aqueous film-forming foams used in firefighting [26]) publications on these systems are rather scarce [27]. Therefore another aim of this work is the study 
of wetting performance of mixtures of fluoro- and hydrocarbon surfactants to provide direct experimental evidence that the synergism in this system is due to their different affinity to the liquid/ air and liquid/hydrocarbon interface.

\section{Materials and Methods}

Fluorosurfactant Zonyl FSN-100 (DuPont), octaethylene glycol monododecylether $\mathrm{C}_{12}(\mathrm{EO})_{8}$ (> $98 \%$, Sigma), trimethoxy(octadecyl)silane (Sigma-Aldrich, >90\%), heptane (HCROMASOLV®, Sigma-Aldrich, >99 \%), ammonia hydroxide solution (Sigma-Aldrich, 28-30 \%) are used as purchased. The aqueous solutions of individual surfactants have been prepared in ultra-pure water produced by Millipore $\mathrm{Q}(15 \mathrm{M} \Omega \mathrm{cm})$ and then mixed in desired proportions.

Both surfactants used below are non-ionic and have similar structure containing ethylene oxide groups. According to [28] chemical composition of Zonyl FSN-100 is $\mathrm{CF}_{3}\left(\mathrm{CF}_{2}\right)_{5}(\mathrm{EO})_{14}$, whereas according to [29] it is $\mathrm{CF}_{3}\left(\mathrm{CF}_{2}\right)_{7.4}(\mathrm{EO})_{13.7}$.

The silanized glass prepared according to protocol proposed in Ref. [30] was used as a substrate for spreading experiments. Microscopic glass slides (Fisher Scientific) were cleaned for 1 hour in concentrated ammonia hydroxide solution, washed in plenty of water in ultrasonic bath, dried in oven at $140{ }^{\circ} \mathrm{C}$ during 1 hour, silanized by immersion in a $5 \mathrm{mM}$ trimethoxy(octadecyl)silane solution in heptane for $24 \mathrm{~h}$, washed 3 times in heptane and dried in oven at $70{ }^{\circ} \mathrm{C}$ during 1 hour. The equilibrium contact angle of water on this substrate was $102 \pm 3^{\circ}$.

Contact angle, radius of spreading, surface and interfacial tension were measured with DSA-100 (Kruss). Surface tension was measured using bubble shape analysis. The interfacial tension at water/heptane interface was measured using heptane drop placed into aqueous solution with v:v ratio about 1000 to prevent surfactant depletion due to partition. Consequent measurements performed for several drops have shown the same value of equilibrium interfacial tension confirming that depletion can be neglected.

All spreading experiments have been performed at room temperature $\mathrm{T}=22 \pm 2^{\circ} \mathrm{C}$ and relative humidity $\mathrm{RH}=40 \pm 5$.

\section{Results and discussion}


The surface tension isotherms of solutions under investigation close to $\mathrm{cmc}$ are presented in Fig. 1. From the isotherm for Zonyl FSN-100 is found $\mathrm{cmc}=0.05 \pm 0.005$ $\mathrm{g} / \mathrm{l}=0.05 \mathrm{mM}$, for $\mathrm{C}_{12}(\mathrm{EO})_{8} \mathrm{cmc}=0.075 \pm 0.005 \mathrm{~g} / \mathrm{l}=0.14 \mathrm{mM}$. The former value is in a very good agreement with that reported for Zonyl in [29]. The latter is a little bit higher than reported for $\mathrm{C}_{12}(E O)_{8}$ in [31], $0.06 \mathrm{~g} / \mathrm{l}$. The difference can be caused by a variation in the samples chemistry, especially taken into account that surfactant was used as purchased in this study, whereas in [31] it was purified. The concentration of solutions used are 0.1-10g/l for $\mathrm{C}_{12}(\mathrm{EO})_{8}$ and 0.5-10 g/l for Zonyl, i.e. they are above the corresponding $\mathrm{cmc}$ values.

Fig. 1 shows that the surface tension of $\mathrm{C}_{12}(\mathrm{EO})_{8}$ is essentially higher than that of Zonyl. At concentrations above cmc surface tension of $\mathrm{C}_{12}(\mathrm{EO})_{8}$ is $\sim 35.3 \mathrm{mN} / \mathrm{m}$ vs $\sim 21.9 \mathrm{mN} / \mathrm{m}$ for Zonyl. Therefore one could expect that wetting properties of Zonil should be superior as related to $\mathrm{C}_{12}(\mathrm{EO})_{8}$. However this is not the case as shown in Fig. 2 and discussed below. The comparison of interfacial tension at the interface with a hydrocarbon (heptane) shows the opposite to the surface tension trend: interfacial tension at concentrations above $\mathrm{cmc}$ is about $3.4 \mathrm{mN} / \mathrm{m}$ for $\mathrm{C}_{12} \mathrm{EO}_{8}$ and $8,5 \mathrm{mN} / \mathrm{m}$ for Zonyl. This difference in the interfacial tension proves that fluorosurfactant adsorbs more readily on water/air interface than on water/hydrocarbon interface.

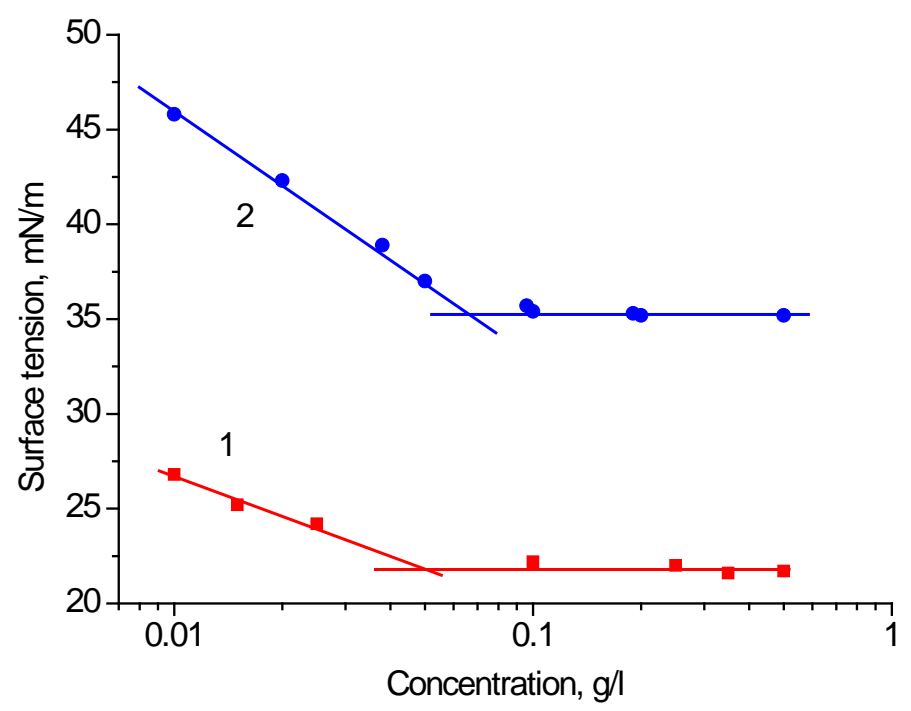

Fig. 1.Surface tension isotherms: 1 - Zonyl FSN-100, $2-\mathrm{C}_{12}(\mathrm{EO})_{8}$. 
The values of equilibrium contact angle for solutions of Zonyl, $\mathrm{C}_{12}(\mathrm{EO})_{8}$ and their mixtures are presented in Fig. 2. The values for pure Zonyl are represented by points corresponding to $\mathrm{c}=0$. It is clear from Fig. 2 that for both pure surfactant solutions contact angle decreases gradually with the increase of concentration (line 1 is a linear fit of the experimental results), the decrease being steeper for Zonyl solutions, for which equilibrium contact angle changes from 57 to $52^{\circ}$ in the studied range of concentrations. For $\mathrm{C}_{12}(\mathrm{EO})_{8}$ equilibrium contact angle is in the range $54-55^{\circ}$, i.e. the considerable difference in the surface tension does not cause a noticeable difference in the equilibrium contact angle values, because it is compensated by the better adsorption of $\mathrm{C}_{12}(\mathrm{EO})_{8}$ on solid/liquid interface.

As it is seen in Fig. 2 the mixtures of considered surfactants demonstrate essential synergism in their wetting performance with equilibrium contact angle for mixtures of optimal composition being 15-20 ${ }^{\circ}$ smaller than that for individual surfactants. Minimum equilibrium contact angle was observed at concentrations $\mathrm{C}_{12}(\mathrm{EO})_{8}$ around half of those of Zonyl. Taking into account the molecular weight of $\mathrm{C}_{12}(\mathrm{EO})_{8}$ $M=538.75 \mathrm{~g} / \mathrm{mol}$ being nearly twice lower than that of Zonyl ( $M=950 \mathrm{~g} / \mathrm{mol}$ according to DuPont information sheet and $\mathrm{M}=1044 \mathrm{~g} / \mathrm{mol}$ according to [29]) it can be concluded that the 1:1 mixtures of surfactant solutions with close molar concentration demonstrates the best wetting properties. 


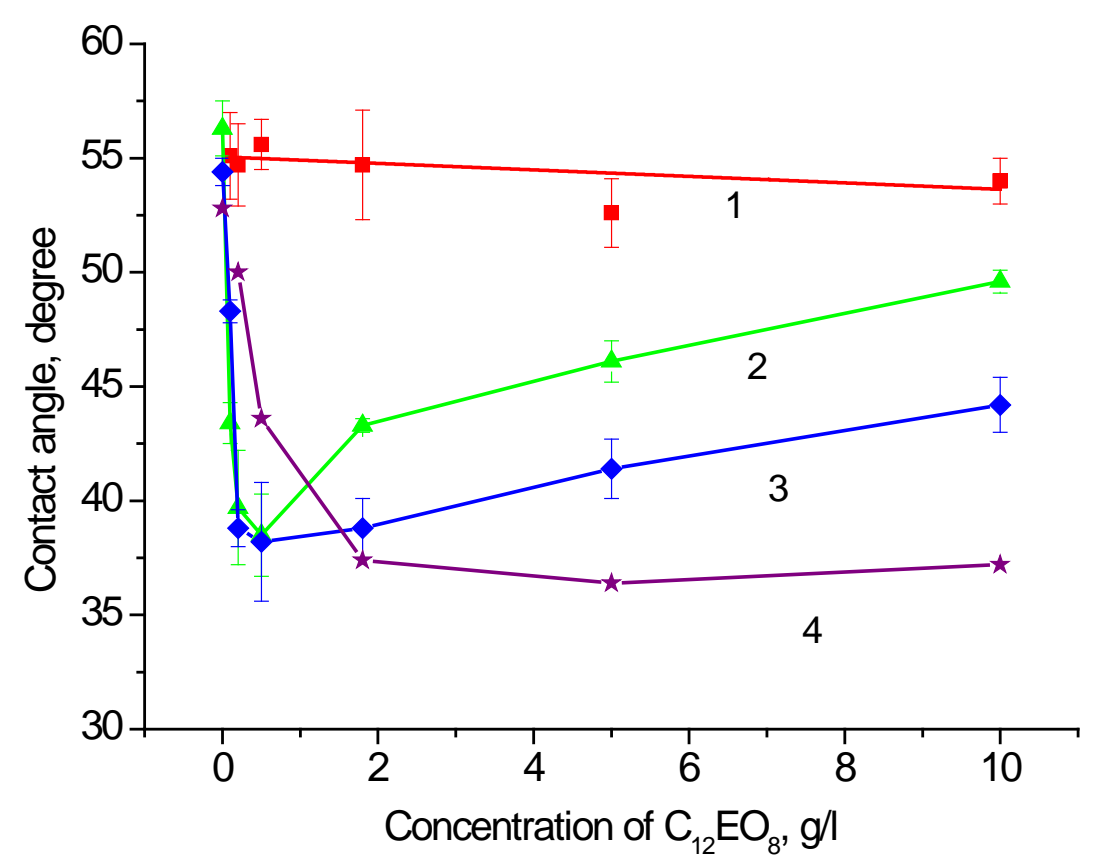

Fig. 2 - contact angle on silanized glass of pure $\mathrm{C}_{12} \mathrm{EO}_{8}$ surfactant solution (1) and v:v=1:1 mixtures with Zonyl FSN-100 solutions of concentration $0.5 \mathrm{~g} / \mathrm{l}(2), 1 \mathrm{~g} / \mathrm{l}(3)$, $10 \mathrm{~g} / \mathrm{l}(4)$.

Let us consider how the spreading performance is related to the surface tension of the mixtures. It is seen from Fig. 3 that addition of $C_{12}(E O)_{8}$ to Zonyl solution results in an increase of surface tension, i.e. it can be assumed that surfactants demonstrate a competitive adsorption at water/air interface and there is no significant interaction between surfactant molecules, resulting in the lowering of surface tension. Comparison of results in Fig. 2 and Fig. 3 shows that despite the increase in the surface tension of mixed solution the contact angle decreases by addition of $\mathrm{C}_{12}(\mathrm{EO})_{8}$ up to molar concentration nearly equal to the concentration of Zonyl solution. The minimum contact angle corresponds to the curve 4 in Fig. 3 . The surface tension of this composition is $\sim 2 \mathrm{mN} / \mathrm{m}$ higher than that of pure Zonyl. It can be therefore assumed that the adsorption at the solid/liquid interface is also competitive and addition of $\mathrm{C}_{12}(\mathrm{EO})_{8}$ results in a decrease of the interfacial tension at solid/liquid interface. By further increase of $\mathrm{C}_{12}(\mathrm{EO})_{8}$ concentration the decrease in 
the solid/liquid interfacial tension does not compensate any more the increase in the surface tension and the contact angle begins to increase.

Note that the synergism in wetting properties demonstrated by the mixtures of hydrocarbon surfactants has a different nature than synergism of mixtures of cationic and anionic surfactants [12-14]. In the latter case the synergism is due to decrease of electrostatic repulsion in the adsorbed monolayer resulting in the considerable decrease of both liquid/air and solid/liquid interfacial tensions [12-14]. In the former case, considered here, synergism is due to preferable adsorption of components of the mixture at one of the interfaces.

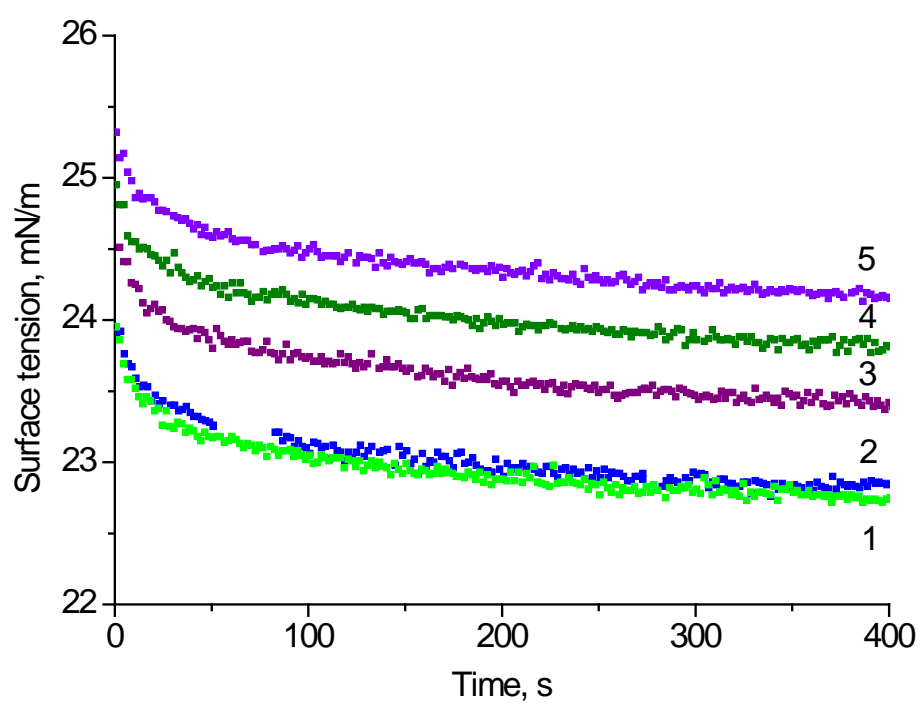

Fig. 3. Dynamic surface tension of $v: v=1: 1$ mixtures of Zonyl FSN-100 solution of concentration $10 \mathrm{~g} / \mathrm{l}$ with $\mathrm{C}_{12} \mathrm{EO}_{8}$ solutions of concentrations $1-0.2 \mathrm{~g} / \mathrm{l}, 2-0.5 \mathrm{~g} / \mathrm{l}, 3$ $-1.8 \mathrm{~g} / \mathrm{l}, 4-5 \mathrm{~g} / \mathrm{l}, 5-10 \mathrm{~g} / \mathrm{l}$.

Fig. 4 shows time of spreading for the solutions presented in Fig. 2, i.e. time when diameter of spreading drops stops to grow. Solutions of individual surfactants reach their maximum diameter faster than mixtures. Their spreading time decreases slightly with an increase of concentration and is directly related to the dynamic surface tension. For example for $1 \mathrm{~g} / \mathrm{l}$ solution of Zonyl $\mathrm{t}_{\mathrm{spr}} \sim 60 \mathrm{~s}$ and the dynamic 
surface tension at this concentration levels off at $\sim 100 \mathrm{~s}$. For $1 \mathrm{~g} / \mathrm{l}$ solution of $\mathrm{C}_{12}(\mathrm{EO})_{8} \mathrm{t}_{\mathrm{spr}} \sim 20 \mathrm{~s}$ and the dynamic surface tension levels off at $\sim 40 \mathrm{~s}$.

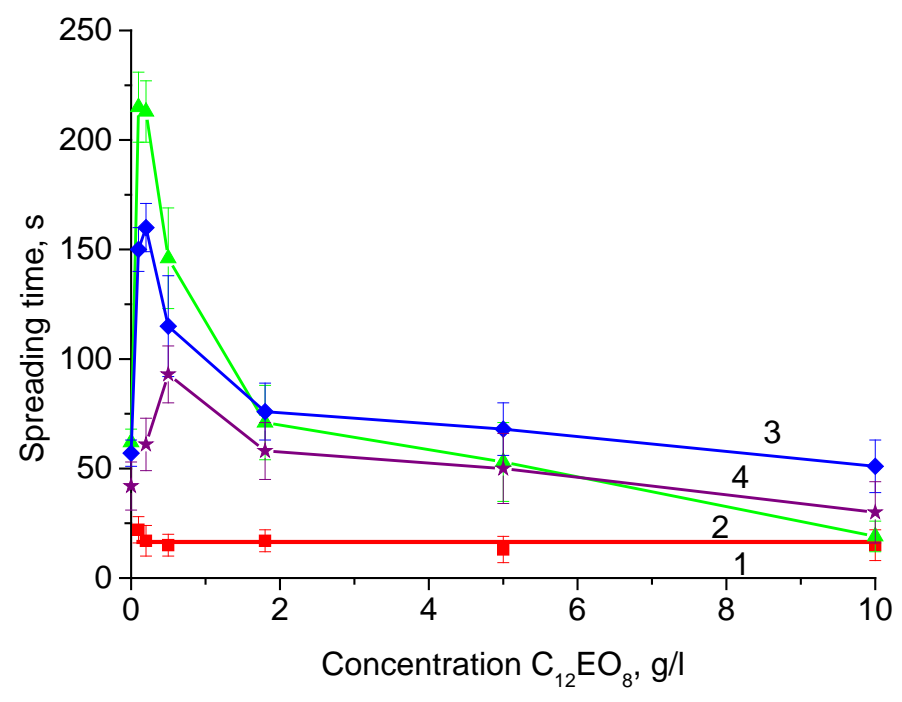

Fig. 4. Time of spreading of pure $\mathrm{C}_{12} \mathrm{EO}_{8}$ surfactant solution (1) and $\mathrm{v}: \mathrm{v}=1: 1$ mixtures with Zonyl FSN-100 solutions of concentration 0.5 g/l (2), 1 g/l (3), 10 g/l (4).

For mixtures with concentration of Zonyl kept constant the spreading time increases with an increase of concentration of $\mathrm{C}_{12} \mathrm{EO}_{8}$ at its small concentrations, but then decreases at larger concentrations. This can be explained by competitive adsorption from the mixture. When concentration of $\mathrm{C}_{12} \mathrm{EO}_{8}$ is small its adsorption is also small and equilibration is determined mainly by adsorption of Zonyl (see curves 1 and 2 in Fig. 3). At higher concentrations adsorption of $\mathrm{C}_{12} \mathrm{EO}_{8}$ increases, but the concentrations is still small and diffusion of $\mathrm{C}_{12} \mathrm{EO}_{8}$ becomes time limiting step. As a result the spreading time increases. The further increase in the concentration of $\mathrm{C}_{12} \mathrm{EO}_{8}$ results in its faster kinetics and therefore in the decrease of the spreading time. In other words, the maximum of curves in Fig. 4 is the result of non-linear dependence of relative adsorption and adsorption kinetics on concentration $\mathrm{C}_{12} \mathrm{EO}_{8}$. 
Note, the maximum of curves in Fig. 4 does not coincide with the minimum contact angle (Fig. 2).

The spreading exponent for individual solutions is presented in Fig. 5. It is much smaller than spreading exponent of pure liquids during viscous stage of spreading $(\alpha \sim 0.1)$. To compare the results of spreading of surfactant solutions with those of pure liquids, the spreading kinetics of silicone oil (viscosity standard $500 \mathrm{mPa} \cdot \mathrm{s}$ ) on Teflon film (equilibrium contact angle of water, $C A W=116 \pm 2^{\circ}$ ) was followed up. The contact angle of the silicone oil on the Teflon film was $\sim 59^{\circ}$, i.e. rather close to contact angle both studied surfactant solutions. Spreading exponent for the silicone oil on Teflon, $\alpha \sim 0.07$ was lower that for the case of complete wetting, but considerably higher than that of individual surfactant solutions. Spreading time was $\sim 3$ s, i.e. much shorter than spreading time of surfactant solutions.

The spreading exponent of mixtures increases with the decrease of the contact angle (Fig. 6) and reaches maximum at the same compositions at which the minimum of the contact angle was observed.

The spreading does not follow the power law presented in Figs. 5 and 6 during the whole time span shown in Fig. 4. It slows down during the final stage of spreading. The duration of stage obeying the power law is shown in Fig. 7.

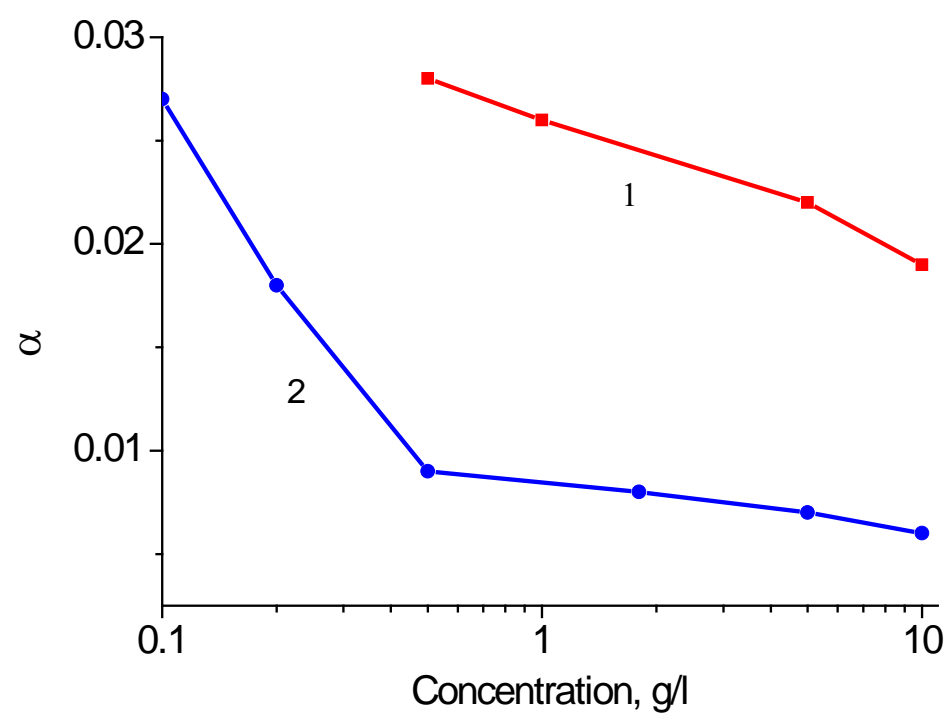


Fig. 5. The spreading exponent $\alpha$ for aqueous solutions: 1 - Zonyl FSN-100, 2 $\mathrm{C}_{12} \mathrm{EO}_{8}$ on sylanized glass.

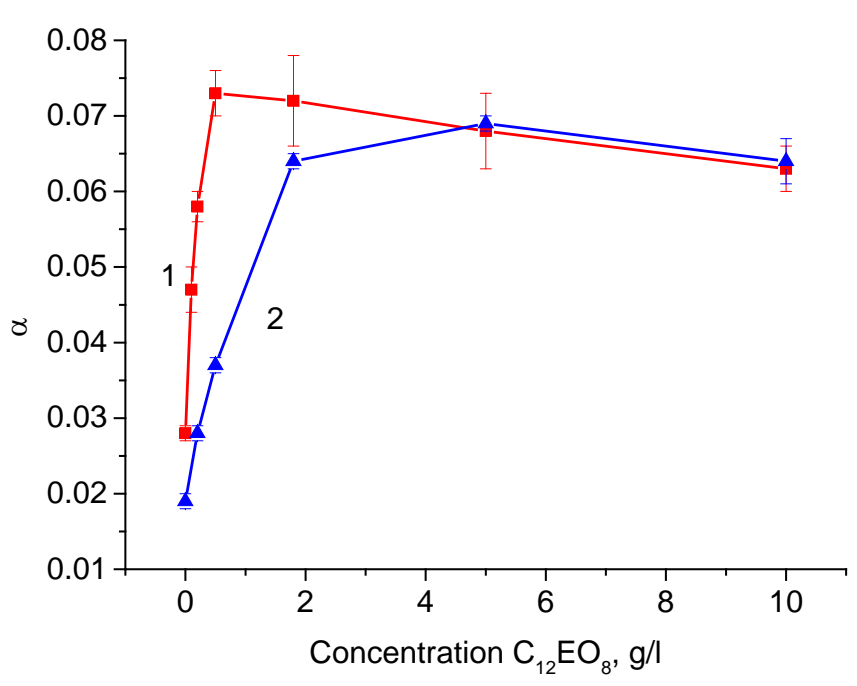

Fig. 6. The spreading exponent, $\alpha$, for mixtures depending on $\mathrm{C}_{12} \mathrm{EO}_{8}$ concentration. Concentration of Zonyl FSN-100: 1 - $0.5 \mathrm{~g} / \mathrm{l}, 2-10 \mathrm{~g} / \mathrm{l}$.

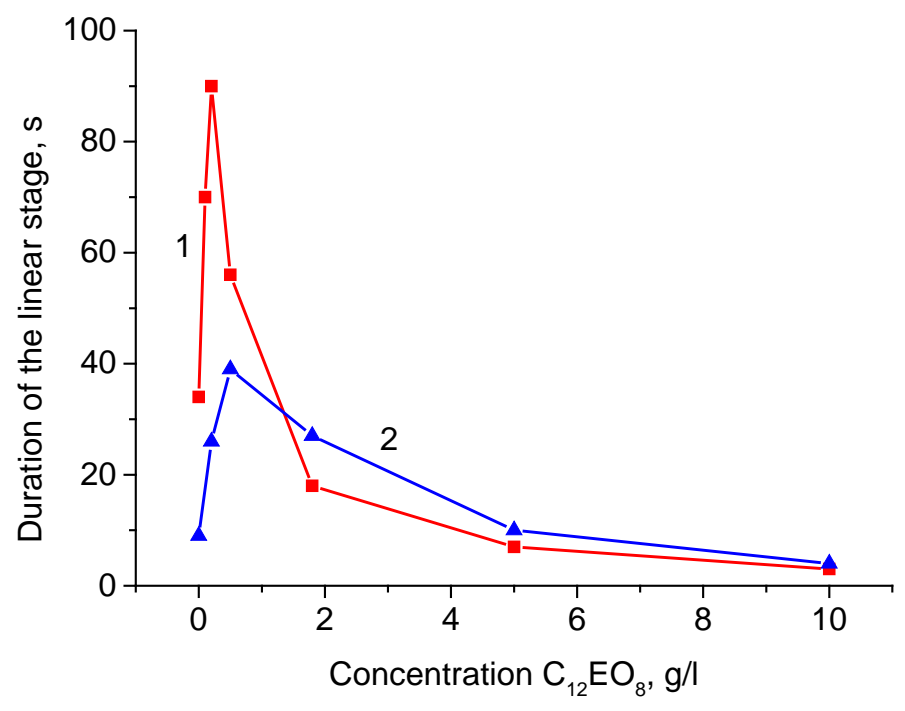

Fig. 7. Duration of the stage obeying the power law with the exponent according to Figs. 5 and 6 depending on $\mathrm{C}_{12} \mathrm{EO}_{8}$ concentration. Concentration of Zonyl FSN-100: $1-0.5 \mathrm{~g} / \mathrm{l}, 2-10 \mathrm{~g} / \mathrm{l}$. 
The special two-step protocol of spreading experiments for mixtures, when first a drop of one of the solutions is placed on the substrate and then a drop of the second one is added on its top was proposed in [12] for catanionic mixtures to suppress the effect of crystallisation. It was however shown in $[13,14]$ that the spreading kinetics does not depend on the protocol (premixed solutions or drop by drop) in the case when concentrations of the components in the mixture are out of the phase separation region. There was no visible phase separation in the mixtures used in this study, but the spreading kinetics depends considerably on the protocol used as shown in Fig. 8.

Premixed solution spreads much faster as compared to the case when one drop is placed on the substrate and second added then on its top. The results of Fig. 8 show clearly the effect of the competitive adsorption. When the drop of Zonyl solution is placed onto the top of $\mathrm{C}_{12} \mathrm{EO}_{8}$ solution it spreads quickly over the liquid surface due to Marangoni flow (the surface tension of Zonyl solution is lower than that of $\mathrm{C}_{12} \mathrm{EO}_{8}$ solution). Therefore in the first moments after Zonyl application, mostly this solution is present at the leading edge of spreading and the spreading diameter of the drop stabilises for some time at the value close to that of pure Zonyl solution. There is some induction time needed for solution of $\mathrm{C}_{12} \mathrm{EO}_{8}$ to diffuse to the contact line region and after that the spreading proceeds further.

If Zonyl solution is applied first and $\mathrm{C}_{12} \mathrm{EO}_{8}$ solution afterwards, there is no essential Marangoni convection, because the surface tension of $\mathrm{C}_{12} \mathrm{EO}_{8}$ solution is higher than that of Zonyl solution. Only gravitational mixing takes palace. Therefore there is some induction time until $\mathrm{C}_{12} \mathrm{EO}_{8}$ reaches solid/ liquid interface and replaces Zonyl.

It is interesting that despite obviously slower spreading by using drop by drop protocol, the spreading exponent is very similar, $0.062 \pm 0.002$ in all three cases, if compare the initial kinetics of spreading for mixed solutions and kinetics inside time span 50-200 s for the drop by drop protocol. Therefore the visible difference in the spreading rate is solely due to the difference in the pre-factor.

Obviously the final contact angle presented by curves 2 and 3 in Fig. 8 is not the equilibrium (advancing) one, as spreading radius is still increasing. However for the 
spreading time larger than 100 s evaporation becomes important. It is seen from Fig. 8 that in the case of premixed solution (curve 1) the spreading diameter reaches maximum inside $100 \mathrm{~s}$ time span. The contact angle decreases after that from the advancing to receding value due to evaporation [32]. Therefore the direct comparison of the advancing contact angles for various protocols is impossible due to higher spreading and therefore noticiable effect of evaporation in the case of drop by drop protocol. However the estimations made under assumption that the decrease in the contact angle due to evaporation is comparable for all three experimental protocols show that the values of equilibrium contact angle should be close to each other.

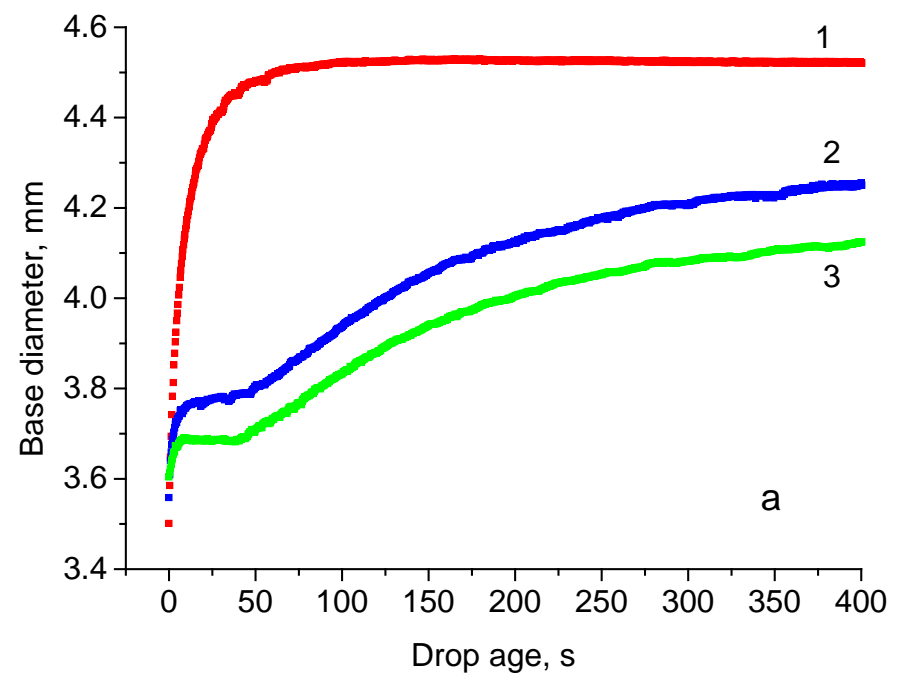




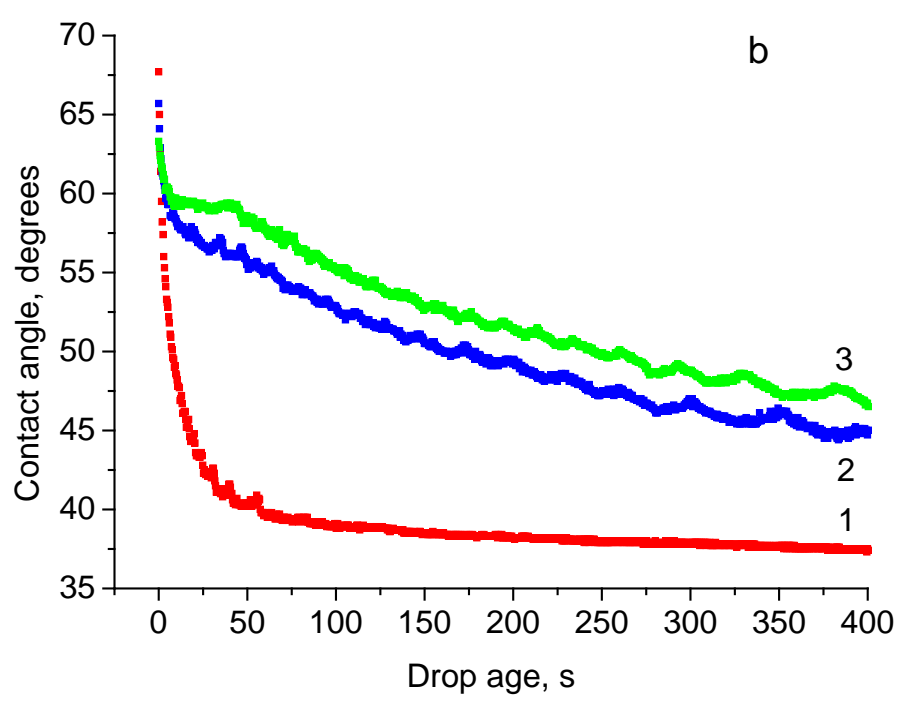

Fig. 8. Spreading kinetics of composition v:v=1:1 $5 \mathrm{~g} / \mathrm{l} \mathrm{C}{ }_{12} \mathrm{EO}_{8}+10 \mathrm{~g} / \mathrm{l}$ Zonyl FSN-100 $\mathrm{a}$ - drop diameter vs time, $\mathrm{b}$ - contact angle vs time: 1 - premixed solution, 2 droplet of Zonyl on the top of droplet of $\mathrm{C}_{12} \mathrm{EO}_{8}, 3$ - droplet of $\mathrm{C}_{12} \mathrm{EO}_{8}$ on the top of droplet of Zonyl.

\section{Conclusions}

Mixed solutions of non-ionic fluoro- and hydrocarbon surfactants demonstrate considerable synergism in the spreading properties: equilibrium contact angle of the mixtures of optimal composition is $15-20^{\circ}$ lower than that of individual surfactants. The synergism is due to different affinities of surfactants to the interfaces involved in spreading. The fluoro-surfactant, Zonyl FSN-100 is more effective at water/air interface lowering the surface tension till $\sim 21.9 \mathrm{mN} / \mathrm{m}$ at concentrations above $\mathrm{cmc}$ as compared with $\sim 35.3 \mathrm{mN} / \mathrm{m}$ for solutions of hydrocarbon surfactant $\mathrm{C}_{12} \mathrm{EO}_{8}$. The opposite trend was observed at water/heptane interface, with interfacial tension being about $3.4 \mathrm{mN} / \mathrm{m}$ for $\mathrm{C}_{12} \mathrm{EO}_{8}$ and $8,5 \mathrm{mN} / \mathrm{m}$ for Zonyl.

The spreading kinetics both individual surfactant solutions and their mixtures obey the power law during nearly of the half of the full spreading time. After that the spreading slows down. The spreading exponent (related to the power law spreading) is smaller than that of pure liquids. The value of the spreading exponent decreases 
with an increase of the equilibrium contact angle. The spreading time correlates with the adsorption kinetics.

Spreading kinetics depend of the experimental protocol: premixed solution spreads faster in comparison with the case when first a drop of one solution is placed on the substrate and then the drop of another solution is applied on the top of the first drop.

\section{Acknowledgments}

This research was supported by Engineering and Physical Sciences Research Council, UK, grant EP/D077869/1; by CoWet Marie Curie, EU project; by European Space Agency, EVAPORATION MAP, FASES and PASTA projects; and by COST MP1106 EU project. We thank Prof. M. Pletnev for fruitful discussions.

\section{References}

[1] Biance A.-L., Clanet C., Quere D. First steps in the spreading of a liquid droplet. Phys. Rev. E 69 (2004) 016301.

[2] Chen L., Bonaccurso E. Effects of surface wettability and liquid viscosity on the dynamic wetting of individual drops. Phys. Rev. E 90 (2014) 022401.

[3] Tanner L.H. The spreading of silicone oil droplets on horizontal surfaces. J. Phys. D: Appl. Phys. 12 (1979) 1473-1484.

[4] Starov V.M., Kalinin V.V., Chen J.D. Spreading of liquid drops over dry surfaces. Adv. Colloid Interface Sci. 50 (1994) 187-221.

[5] Lopez J., Miller C.A., Ruckenstein E. Spreading kinetics of liquid drops on solids.

J. Colloid Interface Sci. 56 (1976) 460-468.

[6] Bird J.C., Mandre S., Stone H.A. Short-time dynamic of partial wetting. Phys. Rev. Lett. 100 (2008) 234501. 
[7] Wang. X., Chen L., Bonaccurso E., Venzmer J. Dynamic wetting of hydrophobic polymers by aqueous surfactant and superspreader solutions. Langmuir, 29 (2013) $14855-14864$.

[8] Ananthapadmanabhan K.P., Goddard E.D., Chandar P. A study of the solution, interfacial and wetting properties of silicone surfactants. Coll. Surf. 44 (1990) 281297.

[9] Zhu S., Miller W.G., Scriven L.E., Davis H.T. Superspreading of water-silicone surfactant on hydrophobic surfaces. Coll. Surf. A, 90 (1994) 63-78.

[10] Stoebe T., Lin Z., Hill R.M. Ward M.D., Davis H.T. Enhanced spreading of aqueous films containing ethoxilated alcohol surfactants on solid substrates. Langmuir 13 (1997) 7270-7275.

[11] Stoebe T., Hill R.M. Ward M.D., Davis H.T. Enhanced spreading of aqueous films containing ionic surfactants on solid substrates. Langmuir 13 (1997) 7276-8281.

[12] Wu Y., Rosen M.J. Synergism in the spreading of hydrocarbon -chain surfactants on polyethylene film - anionic and cationic mixtures by a two-step procedure. Langmuir 21 (2005) 2342-2348.

[13] Kovalchuk N.M., Barton A., Trybala A., Starov V. Surfactant enhanced spreading: catanionic mixture. Colloid Interface Sci. Comm. 1 (2014) 1-5.

[14] Kovalchuk N.M., Barton A., Trybala A., Starov V. Mixtures of catanionic surfactants can be superspreaders: comparison with trisiloxanesuperspreader. J. Colloid Interface Sci. 459 (2015) 250-256.

[15] Hill R.M. Superspreading. Curr. Opinion Colloid Interface Sci. 3 (1998) 247-254. 
[16] Venzmer J. Superspreading - 20 years of physic-chemical research. Curr. Opinion Colloid Interface Sci. 16 (2011) 335-343.

[17] Nikolov A., Wasan D. Superspreading mechanisms: an overview. Eur. Phys. J. Special topics, 197 (2011) 325-341.

[18] Kovalchuk N.M., Trybala A., Arjmandi-Tash O.,Starov V. Surfactant-enhanced spreading: Experimental achievements and possible mechanisms, Adv. Colloid Interface Sci. 2015 doi: http://dx.doi.org/10.1016/j.cis.2015.08.001.

[19] Dutschk V., Sabbatovskiy K.G., Stolz M. Grundke K., Rudoy V.M. Unusual wetting dynamics of aqueous surfactant solutions on polymer surfaces. J. Colloid Interface Sci. 267 (2003) 456-462.

[20] Starov V.M., Kosvintsev S.R., Velarde M.G. Spreading of surfactant solutions over hydrophobic substrates. J. Colloid Interface Sci. 227 (2000) 185-190.

[21] Starov, V. M.; Velarde; M. G.; Radke, C. J. Dynamics of wetting and spreading. Surfactant Sciences Series 138; Taylor and Francis: London, 2007.

[22] Kumar N., Varanasi K., Tilton R.D., Garoff S. Surfactant self-assembly ahead of the contact line on a hydrophobic surface and its implication for wetting. Langmuir 19 (2003) 5366-5373.

[23] Ivanova N., Starov V., Johnson D., Hilal N., Rubio R. Spreading of aqueous solutions of trisiloxanes and conventional surfactants over PTFE AF coated silicone wafers. Langmuir 25 (2009) 3564-3570.

[24] Rosen M.J. Predicting synergism in binary mixtures of surfactants. Progr. Colloid Polymer Sci. 95 (1994) 39-47.

[25] Kovalchuk N.M., Trybala A., Starov V., Matar O, IvanovaN. Fluoro- vs hydrocarbon surfactants: Why do they differ in wetting performance? Adv. Colloid Interface Sci. 2014, 210, 65-71.

[26] Pabon M, Corpart JM, Fluorinated surfactants: synthesis, properties, effluent treatment. J. Fluorine Chem. 114 (2002) 149-156.

[27] Zhao GX, Zhu BY, Spreading of mixed solutions of fluorocarbon and hydrocarbon surfactants on oil. Colloid Polymer Sci. 261 (1983) 89-91. 
[28] Szymczyk, K. The properties of binary mixtures of ethoxilatedoctyl phenols with ethoxilated fluorinated alkanols at the water/air interface. J. Surfact. Deterg. 14 (2011) 415-423.

[29] Skvarla J., Uchman M., Prochazka, K., Tosner, Z., Garamus, V.M., Pispas, S. Stepanek, M. Micellization of Zonyl FSN-100 fluorosurfactant in aqueous solutions Coll. Surf. A 443 (2014) 209-215.

[30] Wenzler L.A., Moyes G.L., Olson L.G., Harris J.M., Beebe T.R., Jr. Singlemolecule bond-rupture force analysis of interactions between AFM tips and substrates modified with organosilanes. Anal. Chem. 69 (1997) 2855-2861.

[31] Rosen M.J., Cohen A.W., Dahanayake M., Hua X. Relationship of structure to properties in surfactants. 10. Surface and thermodynamic properties of 2dodecyloxypoly(ethenoxyethanol)s, $\mathrm{C}_{12} \mathrm{H}_{25}\left(\mathrm{OC}_{2} \mathrm{H}_{2}\right)_{\times} \mathrm{OH}$, in aqueous solution. J. Phys. Chem. 86 (1982) 541-545.

[32]. Semenov S., Trybala A., Rubio R.G., Kovalchuk N., Starov V., Velarde M.G. Simultaneous preading and evaporation: Recent developments. Adv. Coll. Int. Sci. 206 (2014) 382-398. 